\title{
The Q-B Solution
}

Paul T E Cusack*

1641 Sandy Point Rd, Saint John, NB, Canada E2K 5E8, Canada

*Corresponding author: Cusack PTE, Independent Researcher, BSc E, DULE, 1641 Sandy Point Rd, Saint John, NB, Canada E2K 5E8, Canada, Tel: (506) 214-3313; E-mail: St-michael@hotmail.com

Received date: January 17, 2017, Accepted date: February 20, 2017, Published date: February 27, 2017

Copyright: (c) 2017 Cusack PTE. This is an open-access article distributed under the terms of the Creative Commons Attribution License, which permits unrestricted use, distribution, and reproduction in any medium, provided the original author and source are credited.

\section{Abstract}

In this paper, we use Euler's Formula and Astrotheology Physics to determine the mathematical mechanism that may be used by the Fed. Chairman to set interest rates and projected inflation. I call this "Cusack-Bernanke Solution "or the "Q-B Solution."

\section{Keywords}

\section{Introduction}

I expected, the Fed Chair's job is to maintain a straight line between a too hot economy and a too cold one [1,2]. How does he do that? He must keep the pressure on the economy just right. Here's how:

Euler's' Formula:

$\mathrm{e}^{\wedge}(\mathrm{iPi})=-1$

So,

$1 / 2 \mathrm{e}(1-\mathrm{iS})=1 / 2 \mathrm{e}(1+\mathrm{iS})$

We know $\mathrm{i}=0.618$, and cuz $=\mathrm{Pi}-\mathrm{e}=0.4233$

So solving:

$\mathrm{t}(1-0 . \mathrm{i}) \mathrm{S}=(1+\mathrm{i}) \mathrm{S}(\mathrm{t}+\mathrm{dt})$

$(1-0.618) \mathrm{t}=(1+0.618) \mathrm{S}(\mathrm{t}+\mathrm{dt})$

$\mathrm{t}=1.618 / 0.38(\mathrm{t}+\mathrm{dt})$

$\int \mathrm{t} / \mathrm{dt}=\int 4.23$

$\mathrm{t}^{2}=4.23 \mathrm{t}$

$\mathrm{t}=4.23$

$\mathrm{t}=67.35 \% \sim 2 / 3$

For a full economic cycle $=2 \pi$

$\mathrm{t}(4.23(\mathrm{t}+\mathrm{dt})$

$2 / 3=4.23(2 / 3)+4.23 \mathrm{dt}$

$3.23=6.28 \mathrm{dt}$

$\mathrm{dt}=2 \pi / 3.23$

$\mathrm{dt}=6.28 / 3.28 \sim 2$

$\mathrm{t}-\mathrm{dt}=2 / 3+2=0.2666=\mathrm{F}$ Force

$\mathrm{F}=\mathrm{Ma}=0.2666=\mathrm{M}(0.8415)$

$\mathrm{M}=0.318=1 / \pi$

Maximum Output $=0.8415=81.45=\sin 45^{\circ}=\cos 45 \circ=\mathrm{a}=\mathrm{v}$

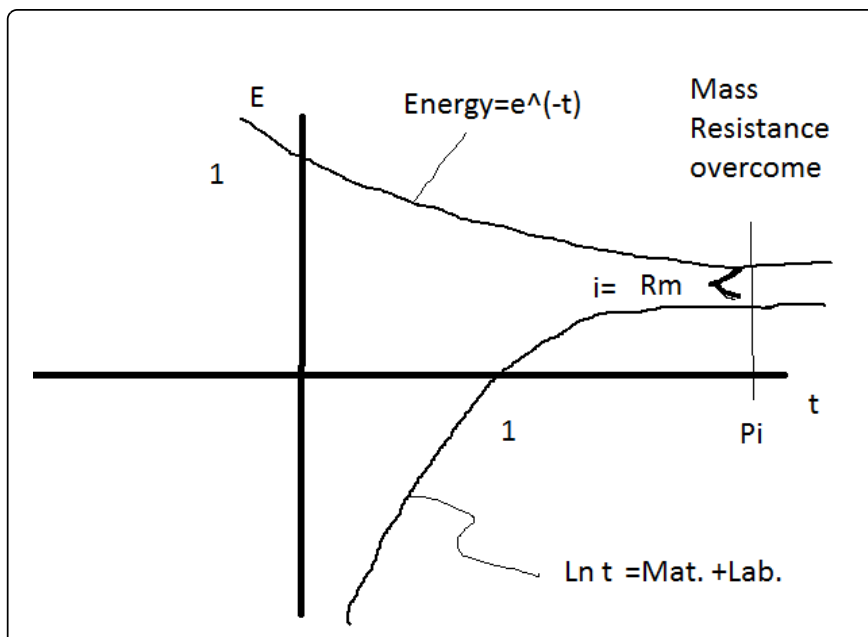

Figure 1: Graphical representation of the balance between too much pressure and too little.

Work $\mathrm{W}=\mathrm{F}^{\star} \mathrm{d}$

Energy $\mathrm{E}=\mathrm{W}=\mathrm{M} \$=$ Money Supply

Balance between too much pressure and too little,

$\operatorname{Sin} 45=\cos 45$

$\mathrm{E}=1 / \mathrm{sqrt} 2$

We know from basic physics:

$\mathrm{D}=\mathrm{vit}+1 / 2$ at ${ }^{\wedge} 2$

$0.265=0+1 / 2(0.707) \mathrm{t}^{\wedge} 2$

$\mathrm{T}=0.866=\sin 60$ degrees

Work=stored money $=\mathrm{M} \$$

$\mathrm{M} \$=1 / \mathrm{t}^{\star} 1 / \mathrm{t}$

$\mathrm{M} \$ /$ frequency $=1 / \mathrm{t}$

$=1 / t^{\wedge} 2$ 


$$
\begin{aligned}
& \mathrm{T}=0.1 / 0.75=4 / 3=0.1334 \\
& \mathrm{M} \$=0.4244 \sim(\mathrm{Pi}-\mathrm{e})=\mathrm{cuz} \\
& \mathrm{M} \$=0.4233 \%
\end{aligned}
$$

Now, if $i=0.04244$, And Inflation $=2 \%$,

Real rate $\mathrm{i}=0.04244-0.02=0.0224$ Or $2.214 \%$

Plugging this into the Golden Mean Parabola,

$\mathrm{T}^{\wedge} 2-\mathrm{t}-1=\mathrm{E}$

$(2.24)^{\wedge} 2-2.24-1=1.777=$ sqrt $\mathrm{Pi}$

Volume of a sphere $=4 / 3$ Pi $\mathrm{R}^{\wedge} 3$

$\mathrm{Vol}=\mathrm{E}=0.4244$

$4 / 3 \mathrm{Pi}^{*} \mathrm{R}^{\wedge} 3=0.4244$

$\mathrm{R}=2.14$

$=1-\mathrm{Pi}$

Area $=\mathrm{PiR}^{\wedge} 2$

$=\mathrm{Pi}^{\star} 2.14^{\wedge} 2=14.38$

$1-\mathrm{R} / 100=58.8$ degrees $=\sim 1 \mathrm{rad}$

Coreference $=2 \mathrm{PiR}$

$=2 \mathrm{Pi}^{*}(2.14)$

$=13.43$

$1=13.43 / 100=0.866=\sin 60$ degrees

$=$ Supe force

Considering the GDP Equation:

$\mathrm{GD}=\mathrm{Y}=\mathrm{G}+\mathrm{I}+\mathrm{C}-\mathrm{S}$

Ln $\mathrm{PI}=17 \%+4.4244 \%+\mathrm{C}-(1 / 7) \%$

$1.1447=6.95 \%+\mathrm{C}$

$\mathrm{C}=1+(1 / 7)$

$=7.52 \%$

So, again,

\author{
$\mathrm{Y}=\mathrm{G}=\mathrm{I}+\mathrm{C}=\mathrm{S}$ \\ $\mathrm{Y}-\mathrm{C}-\mathrm{I}=\mathrm{C}-\mathrm{S}$ \\ $=1+(1 / 7)+1 / 7$ \\ $\mathrm{I}=1.686$
}

But $\mathrm{I}=4.244 \%$

So, $\$ .244 \%-1.686 \%=2.55 \%$

$=$ Inflation

The Optimum Period $\mathrm{T}=1 / \mathrm{t}=0.251$

$\mathrm{LE}=1 / \mathrm{t}=\mathrm{T}$

Ln $\mathrm{T}=\mathrm{Ln} 0.251)=1386$

$1-0 ., 1386=0.863 \sim 0.866=\sin 60$ degrees

$=$ Superforce $=\mathrm{M} \$$

This is the pressure that the Fed Chair must keep on the economy.

So, ideally, the Money Supply should follow the sine curve over the economic cycle [3]. The influence of the combined affect of government spending, $G$ and controlling the money supply, M\$, should sum to a sine wave always.

We'll call it the $\mathrm{Q} / \mathrm{B}=\mathrm{Q}$ uarter Back Solution (Figure 1).

\section{Conclusion}

So, the job of maintaining a constant, maximum growth of the national economy should follow the sine wave.

\section{References}

1. Cusack P (2016) Why the 7 Year Economic Cycle? Boom, Stabilization, Recession, Depression. Journal of Statistics and Mathematical Sciences 2: 53-54.

2. Cusack P (2016) Astro-Theology, Cusack's Universe. Journal of Physical Mathematics 7: 1-8.

3. Cusack P (2016) What is the value of the SQRT (-1)? Journal of Statistics and mathematical Sciences 2: 53-54. 